\title{
Mesenchymal stem cell-conditioned media suppresses inflammation-associated overproliferation of pulmonary artery smooth muscle cells in a rat model of pulmonary hypertension
}

\author{
JUNFENG LIU ${ }^{1-3}$, ZHIBO HAN ${ }^{4}$, ZHONGCHAO HAN ${ }^{4}$ and ZHIXU HE ${ }^{1,2}$ \\ ${ }^{1}$ Laboratory of Tissue Engineering and Stem Cells, Guiyang Medical College; \\ ${ }^{2}$ Department of Pediatrics, Affiliated Hospital of Guiyang Medical College, Guiyang, Guizhou 550004; \\ ${ }^{3}$ Department of Pediatrics, The General Hospital of Huabei Oil Field Company, Renqiu, Hebei 062552; \\ ${ }^{4}$ National Engineering Research Center of Cell Products, AmCellGene Co., Ltd., Tianjin 300457, P.R. China
}

Received January 16, 2015; Accepted November 25, 2015

DOI: $10.3892 /$ etm.2015.2953

\begin{abstract}
Inflammation-associated overproliferation of pulmonary artery smooth muscle cells (PASMCs) is considered to be involved in the pathogenesis of pulmonary hypertension $(\mathrm{PH})$. The administration of mesenchymal stem cell-conditioned media (MSC-CM) has displayed benefits in the treatment of PH, however, the exact mechanism has yet to be elucidated. The present study aimed to determine whether MSC-CM is able to suppress overproliferation of PASMCs in $\mathrm{PH}$ via immunoregulation. By the administration of MSC-CM to monocrotaline (MCT)-induced PH rats, and the development of an in vitro co-culture system comprised of PASMCs and activated $\mathrm{T}$ cells, the therapeutic effects of MSC-CM on $\mathrm{PH}$, and the changes in the expression of correlated factors, including TNF- $\alpha$, calcineurin $(\mathrm{CaN})$ and nuclear factor of activated $\mathrm{T}$ cells (NFAT), were assessed. Immunohistochemical staining results indicated that MSC-CM was able to significantly suppress the production of TNF- $\alpha$ in MCT-induced PH and co-culture systems; and reverse transcription-quantitative polymerase chain reaction results showed significant downregulation of the expression of CaN and NFATc2 in PASMCs $(\mathrm{P}<0.01)$. Furthermore, MSC-CM was able to significantly suppress $\mathrm{CaN}$ activity and NFATc2 activation $(\mathrm{P}<0.01)$, thus inhibiting the overproliferation of PASMCs. Finally, MSC-CM improved abnormalities in hemodynamics and pulmonary
\end{abstract}

Correspondence to: Professor Zhixu He, Laboratory of Tissue Engineering and Stem Cells, Guiyang Medical College, 4 Beijing Road, Guiyang, Guizhou 550004, P.R. China

E-mail:zhixu_he@126.com

Professor Zhongchao Han, National Engineering Research Center of Cell Products, AmCellGene Co. Ltd, 4th Street TEDA, Tianjin 300457, P.R. China

E-mail: hanzhongchao@hotmail.com

Key words: pulmonary hypertension, rat, tumor necrosis factor- $\alpha$, calcineurin, nuclear factor of activated $\mathrm{T}$ cells, mesenchymal stem cells, conditioned media histology in MCT-induced PH. In conclusion, the findings of the current study suggest that administration of MSC-CM has the potential to suppress inflammation-associated overproliferation of PASMCs due to its immunosuppressive effects in $\mathrm{PH}$ and, thus, may serve as a beneficial therapeutic strategy.

\section{Introduction}

Pulmonary hypertension $(\mathrm{PH})$ is a type of lung disease that is severely detrimental to human health, and is often characterized by a progressive increase in pulmonary arterial pressure, eventually resulting in right ventricular overload and finally, heart failure (1). Despite current treatments, including the use of targeted medicine such as sildenafil, assisting in relief of the clinical symptoms of $\mathrm{PH}$, the long-term prognosis remains poor (2). For example, the 5-year survival rate of cystic fibrosis patients with $\mathrm{PH}$ is $40.8 \%$ (3); however, even following lung transplantation, the median survival rate is only $\sim 7$ years (4). Therefore, there is an urgent requirement for the development of more effective therapeutic agents for the treatment of $\mathrm{PH}$.

Numerous studies have served to elucidate the mechanism of $\mathrm{PH}$, highlighting the importance of inflammation and the calcineurin $(\mathrm{CaN}) /$ nuclear factor of activated $\mathrm{T}$ cells (NFAT) signaling pathway (5-13). It has been determined in experimental animal models and in human patients that inflammatory cells are present in the region of remodeled pulmonary arteries (5-8). Furthermore, it has been explicated that the perivascular accumulation of inflammatory cells is essential to pulmonary vascular remodeling (9). Therefore, elevated production of inflammatory cytokines has the potential to be utilized as a predictive marker of survival in patients with PH (9). It remains uncertain as to whether inflammation initiates pulmonary vascular remodeling, or whether it is a bystander effect. However, it is clear that inflammation and associated inflammatory cytokines are involved in the progression of $\mathrm{PH}$. Of the various inflammatory cytokines, the role of tumor necrosis factor- $\alpha$ (TNF- $\alpha$ ) in the overproliferation of pulmonary artery smooth muscle cells (PASMCs) and in vascular remodeling, appears to be significant. TNF- $\alpha$ has the ability to cause calcium influx and promote the proliferation of 
SMCs (10); the activation of the CaN/NFAT signaling pathway is a crucial step within this process (11-13). Therefore, we hypothesize that inflammation-associated overproliferation of PASMCs is a therapeutic target in the treatment of $\mathrm{PH}$.

Mesenchymal stem cells (MSCs), as primitive cells capable of multilineage differentiation and self-renewal, have been observed to effectively inhibit the production of inflammatory cytokines (14), and improve hypoxia-induced $\mathrm{PH}$ in experimental animal models (15). However, there are a number ethical factors that may serve to impede the progress of MSCs into the clinic. Our previous study indicated that the immunosuppressive effect of MSCs are strongly associated with their ability to express immuno-regulatory cytokines and secrete prostaglandin E2 (PGE2) (14). It was also revealed that conditioned media from MSCs could produce cytoprotective effects (15). However, the mechanism by which MSC-conditioned media (CM) suppresses the inflammation-associated overproliferation of PASMCs remains unknown.

In the present study, a rat model of $\mathrm{PH}$ and a co-culture system comprised of PASMCs and activated T cells were used to assess the ability of MSC-CM to suppress the overproliferation of PASMCs. Associated factors, including the expression levels of TNF- $\alpha$ in the rat model and co-culture system, and the expression levels and activation of CaN/NFAT in PASMCs, were also evaluated, in order to better understand the mechanism by which MSC-CM leads to the overproliferation of PASMCs in PH.

\section{Materials and methods}

Preparation of MSC-CM. All studies were approved by the Institutional Review Board of Guiyang Medical College (Guiyang, China) and written informed consent was obtained from all donors. In June 2013, 3 umbilical cords were obtained following normal deliveries from healthy, post-partum women (age, 26-30 years; gestational age, 37-42 weeks) at the Tianjin Central Hospital of Gynecology and Obstetrics (Nankai, China). From these, human umbilical cord-derived MSCs were isolated and cultured in Dulbecco's modified Eagle's medium (DMEM)/F-12 medium (Gibco; Thermo Fisher Scientific, Inc., Waltham, MA, USA) supplemented with $10 \%$ fetal bovine serum (FBS; GE Healthcare Life Sciences, Logan, UT, USA) and Gibco penicillin-streptomycin (100 U/ml; Thermo Fisher Scientific, Inc.), as described in a previous protocol (16). MSCs were identified by analyzing the expression of cell-surface markers or internal cell markers was assessed using a BD FACSCalibur flow cytometer (BD Biosciences, Franklin Lakes, NJ, USA), including cluster of differentiation (CD)11b, CD29, CD34, CD44, CD45, CD54, CD73, CD80, CD86, CD90, CD105, human leukocyte antigen (HLA)-DR, HLA-ABC, Nestin and SRY box-2 (Sox-2). The osteogenic and adipogenic differentiation in vitro were also assessed (16). Briefly, MSCs were seeded in 24-well plates at a density of $10^{4}$ cells $/ \mathrm{ml}$ ( $1 \mathrm{ml} /$ well); after $24 \mathrm{~h}$ of culture, the medium was replaced with osteogenic or adipogenic induction medium. For osteogenic induction, this medium consisted of DMEM/F-12 medium supplemented with 10\% FBS, $100 \mathrm{nmol} / \mathrm{l}$ dexamethasone, $10 \mathrm{mmol} / 1 \beta$-glycerophosphate and $0.2 \mathrm{mmol} / 1$ L-ascorbic acid-2-phosphate (all Sigma-Aldrich, St. Louis, MO, USA). For adipogenic induction, the medium consisted of DMEM/F-12 medium supplemented with $10 \% \mathrm{FBS}, 5 \mathrm{~g} / \mathrm{ml}$ insulin, $1 \mathrm{mmol} / 1$ dexamethasone, $60 \mathrm{mmol} / 1$ indomethacin, and $0.5 \mathrm{mmol} / 1$ isobutylmethylxanthine (all Sigma-Aldrich). After 2 weeks of inducted culture, osteogenic and adipogenic differentiation were identified using Alizarin Red S and Oil Red O stain (Sigma-Aldrich), respectively. MSCs passaged 8-10 times were washed thoroughly with phosphate-buffered saline (PBS; BD Biosciences) and incubated in new medium for $24 \mathrm{~h}$. The MSC-CM was collected by centrifugation at $4{ }^{\circ} \mathrm{C}$, at $2,000 \times \mathrm{g}$ for $10 \mathrm{~min}$, then stored at $-80^{\circ} \mathrm{C}$. For administration to rats, MSC-CM prepared according to the aforementioned protocol was replaced with serum-free TheraPEAK MSCGM-CD medium (Lonza Group Ltd., Basel, Switzerland) at passage 3 .

Experimental animals. All animal studies were approved by the Institutional Animal Care and Use Committee of Guiyang Medical College. Female Sprague-Dawley (SD) rats (age, 8-10 weeks; $\mathrm{n}=18$ ) with body weights of $\sim 200 \mathrm{~g}$ were purchased from and housed in specific pathogen-free units of the Laboratory Animals Center at Tianjin Blood Diseases Hospital (Tianjin, China). The rats were maintained at $25^{\circ} \mathrm{C}$, a relative humidity of $70 \%$ and with a 12-h light/dark cycle.

The rats were randomly divided into three equal groups ( $n=6$ per group), as follows: A PH model group, a MSC-CM administration group and a control group. The PH model was induced by a single subcutaneous injection with monocrotaline (MCT; $60 \mathrm{mg} / \mathrm{kg}$; Sigma-Aldrich), in accordance with a previous study (17). On days 5-9 after injection with MCT, $500 \mu \mathrm{l}$ serum-free MSCGM-CD was subcutaneously injected into the MSC-CM group. The control group was injected with $500 \mu \mathrm{l}$ PBS alone. Rats were anesthetized by intraperitoneal injection of pentobarbital (50 mg/kg; Sigma-Aldrich) 21 days after administration, and right ventricular systolic pressure (RVSP) and mean aortic pressure (MAoP) were determined, according the protocol detailed in a previous study (18).

Subsequent to the aforementioned procedures, rats were sacrificed by decapitation, lung tissues were removed and fixed in $10 \%$ paraformaldehyde at room temperature for $24 \mathrm{~h}$. Serial sections $(5 \mu \mathrm{m})$ were stained with hematoxylin and eosin (Yuanmu Biotechnology Co., Ltd., Shanghai, China), and the medial wall thickness (WT) of pulmonary arterioles was observed under an Olympus BX53 microscope (Olympus Corporation, Tokyo, Japan) and expressed as: $\mathrm{WT}(\%)=[($ medial thickness $\times 2) /$ external diameter $] \times 100$ (19).

Immunohistochemical staining for TNF- $\alpha$ in lung tissue. Serial sections $(5 \mu \mathrm{m})$ were fixed on gelatin-coated slides. Following deparaffinization with two changes of xylene, rehydration with graded ethanol and sequential incubation for $5 \mathrm{~min}$ at room temperature with $0.3 \%$ Triton X-100 (Sigma-Aldrich) and 3\% hydrogen peroxide (Santa Cruz Biotechnology, Inc., Dallas, TX, USA), the sections were incubated with goat polyclonal primary antibody against TNF- $\alpha$ (1:400 dilution; cat. no. sc-1350; Santa Cruz Biotechnology, Inc.) for $12 \mathrm{~h}$ at $4^{\circ} \mathrm{C}$. Following three washes with PBS, the sections were incubated for $30 \mathrm{~min}$ at room temperature with biotinylated rabbit anti-goat monoclonal antibody (1:100 dilution; cat. no. BA-1006; Wuhan Boster Biological Technology, Ltd., Wuhan, China), and the immunoreactivity detected with 
Table I. Rat gene primers for RT-qPCR.

\begin{tabular}{ll}
\hline Primer & \multicolumn{1}{c}{ Sequence } \\
\hline GAPDH F & 5'-CCATTCTTCCACCTTTGATGCT-3' \\
GAPDH R & 5'-TGTTGCTGTAGCCATATTCATTGT-3' \\
CaN F & 5'-CAGAGGGTGCTTCGATTCTC-3' \\
CaN R & 5'-CCCCTAAGAAGAGGTAGCGA-3' \\
NFATc2 F & 5'-CAGCAGATTTGGGAGATGGAAG-3' \\
NFATc2 R & 5'-GACTGGGTGGTAAGTAAAGTGC-3'
\end{tabular}

F, forward; R, reverse; CaN, calcineurin; NFATc2, nuclear factor of activated $\mathrm{T}$ cells $\mathrm{c} 2$; RT-qPCR, reverse transcription-quantitative polymerase chain reaction.

a 3-amino-9-ethylcarbazole peroxidase substrate kit (Wuhan Boster Biological Technology, Ltd.). The sections were counterstained with hematoxylin, and observed under the Olympus BX53 microscope. Mean optical density (OD) was subsequently calculated using Image-Pro Plus Software 6.0 (Media Cybernetics, Rockville, MD, USA).

Isolation of PASMCs and T cells. A total of $4 \mathrm{SD}$ rats with body weights of $\sim 100 \mathrm{~g}$ were sacrificed by decapitation prior to harvesting of pulmonary arteries for PASMC culture using the tissue explants method (20). DMEM/F-12 media supplemented with $10 \%$ FBS and $100 \mathrm{U} / \mathrm{ml}$ penicillin-streptomycin was used. PASMCs were identified by immunofluorescent staining for $\alpha$-smooth muscle actin as follows: PASMCs were sequentially fixed with $4 \%$ paraformaldehyde for $15 \mathrm{~min}$, incubated with $0.3 \%$ Triton X-100 and $1 \%$ bovine serum albumin for $20 \mathrm{~min}$ at room temperature and incubated with rabbit $\alpha$-smooth muscle actin polyclonal antibody ( $\alpha$-SMA; 1:100 dilution; cat. no. 14395-1-AP; ProteinTech Group, Inc., Chicago, IL, USA) overnight at $4^{\circ} \mathrm{C}$. Subsequent to washing with PBS, cells were incubated with fluorescein isothiocyanate-conjugated goat anti-rabbit immunoglobulin $\mathrm{G}$ (1:100 dilution; cat. no. SA00003-2; ProteinTech Group, Inc.) for $2 \mathrm{~h}$ at room temperature in the dark. Finally, cells were incubated with DAPI $(1 \mu \mathrm{g} / \mathrm{ml})$ for $5 \mathrm{sec}$. To extract T cells, the spleens were harvested and $\mathrm{T}$ cells were isolated contingent on their non-adherence to nylon wool (21) and stored at $-80^{\circ} \mathrm{C}$ prior to use.

Co-culture of PASMCs and T cells. The cells were divided into three groups, as follows: Group A (PASMCs alone), Group B (PASMCs + T cells) and Group C (PASMCs + $\mathrm{T}$ cells + MSC-CM). All cells were maintained at $37^{\circ} \mathrm{C}$ in a humidified environment with $5 \% \mathrm{CO}_{2}$. PASMCs at passage 5 were seeded into 24-well plates at a density of $10^{4}$ cells $/ \mathrm{ml}$ $(1 \mathrm{ml} /$ well). In Group A, the media was renewed after $24 \mathrm{~h}$ of culture. In Group B, $0.9 \mathrm{ml}$ of medium was removed, and $0.9 \mathrm{ml} \mathrm{T}$ cell suspension at a density of $10^{5}$ cells $/ \mathrm{ml}$ was added and stimulated by concanavalin A (ConA; $10 \mu \mathrm{g} / \mathrm{ml}$; Sigma-Aldrich). For Group C, the total volume of supernatant was removed and ConA-stimulated $\mathrm{T}$ cells were added at the same density as those in Group B. Furthermore, an additional $0.1 \mathrm{ml}$ MSC-CM was added.
TNF- $\alpha$ protein expression levels in co-culture systems. Following 3 days of co-culture, the cell-free supernatant was collected from each cell group by centrifugation $\left(4^{\circ} \mathrm{C}\right.$, $2,000 \times \mathrm{g}, 10 \mathrm{~min}$ ), and expression levels of TNF- $\alpha$ were measured using a Rat TNF- $\alpha$ Mini ABTS ELISA Development kit (PeproTech, Inc., Rocky Hill, NJ, USA), according to the manufacturer's protocol.

Proliferation of PASMCs. Following collection of the supernatant from all three groups, $T$ cells were removed by washing three times with PBS. Next, PASMCs were incubated for $4 \mathrm{~h}$ in $300 \mu 1$ new medium containing $15 \mu 1$ 3-(4, 5-diethylthiazol-2 -yl)-5-(3-carboxymethoxyphenyl)-2-(4-sulfophenyl)-2H-etrazolium, inner salt (MTS; Promega Corporation, Madison, WI, USA) at $37^{\circ} \mathrm{C} / 5 \% \mathrm{CO}_{2}$. The OD of the supernatant was read at $490 \mathrm{~nm}$ using a Model 680 microplate reader (Bio-Rad Laboratories, Inc., Hercules, CA, USA).

mRNA expression of CaN and NFATc2. After 3 days of co-culture, total RNA of PASMCs was extracted from each of the three groups using an E.Z.N.A. Total RNA I kit (Omega Biotek, Inc., Norcross, GA, USA), and reverse transcribed to cDNA using a Moloney Murine Leukemia Virus Reverse Transcriptase kit (Invitrogen; Thermo Fisher Scientific, Inc.). Following this, the cDNA was amplified and the expression of $\mathrm{CaN}$ and NFATc2 were analyzed by quantitative PCR (qPCR) using an Applied Biosystems 7300 Real-Time PCR System with Invitrogen Platinum SYBR Green qPCR SuperMix-UDG w/ROX (Thermo Fisher Scientific, Inc.). The PCR cycling conditions were as follows: $95^{\circ} \mathrm{C}$ for $2 \mathrm{~min}, 95^{\circ} \mathrm{C}$ for $15 \mathrm{sec}$ and $60^{\circ} \mathrm{C}$ for $30 \mathrm{sec}$; this was repeated for 40 cycles, as per our previous study (22). Relative expression levels were quantified by the $2^{-\Delta \Delta \mathrm{Cq}}$ normalization method (23). The primers used are listed in Table I.

CaN activity. PASMCs from each of the three groups were lysed in $1 \mathrm{ml}$ lysate buffer composed of $50 \mathrm{mmol} / 1$ Tris, $0.5 \mathrm{mmol} / 1 \mathrm{DTT}, 50 \mu \mathrm{g} / \mathrm{ml}$ PMSF, $50 \mu \mathrm{g} / \mathrm{ml}$ soybean trypsin inhibitor, $5 \mu \mathrm{g} / \mathrm{ml}$ leupeptin and $5 \mu \mathrm{g} / \mathrm{ml}$ aprotinin (Beijing Dingguo Changsheng Biotechnology Co., Ltd., Beijing, China). Following three freeze-thaw cycles, the supernatant was collected by centrifugation $\left(4^{\circ} \mathrm{C}, 2,000 \mathrm{x} \mathrm{g}, 10 \mathrm{~min}\right)$ for measurement of $\mathrm{CaN}$ phosphatase activity using a Calcineurin assay kit (Nanjing Jiancheng Bioengineering Institute, Nanjing, China).

NFATc 2 activation. After 3 days of co-culture, PASMCs from each of the three groups were sequentially fixed with $4 \%$ paraformaldehyde (15 min), incubated with $0.3 \%$ Triton X-100 and $1 \%$ bovine serum albumin $(20 \mathrm{~min})$, and then incubated with mouse anti-rat NFATc2 monoclonal antibody (1:100 dilution; cat. no. NB300-504; Novus Biologicals, LLC, Littleton, CO, USA) overnight at $4^{\circ} \mathrm{C}$. Subsequent to washing with PBS, cells were incubated with fluorescein isothiocyanate-conjugated goat anti-mouse immunoglobulin G (1:100 dilution; cat. no. SA00003-1; ProteinTech Group, Inc.) for $2 \mathrm{~h}$ at room temperature in the dark. Finally, cells were incubated with DAPI $(1 \mu \mathrm{g} / \mathrm{ml})$ for $5 \mathrm{sec}$ and assessed with a TCS SP5 confocal laser scanning microscope (Leica Microsystems GmbH, Wetzlar, Germany). 
Statistical analysis. Data are presented as mean \pm standard deviation and SPSS software (version 17.0; SPSS, Inc., Chicago, IL, USA) was used for statistical analysis. Differences were compared using an independent-samples t-test or one-way analysis of variance. $\mathrm{P}<0.05$ was considered to indicate a statistically significant difference.

\section{Results}

Identification of MSCs and PASMCs. Human MSCs grown in the serum-free medium presented with a typical fibroblastic shape (Fig. 1A), whilst those cultured in the specific conditioned medium displayed osteogenic (Fig. 1B) and adipogenic (Fig. 1C) differentiation. The MSCs were positive for HLA-ABC (99.73\%), Nestin (68.39\%), Sox-2 (78.46\%), CD29 (100\%), CD44 (100\%), CD54 (86.98\%), CD73 (99.79\%), CD90 (100\%) and CD105 (97.33\%), and negative for CD34, CD45, CD80, CD86, HLA-DR and CD11b (Fig. 1D).

After 5 days of culture using the tissue explants method, PASMCs harvested from rats were observed surrounding the tissue explants (Fig. 1E). Following a further 3 passages of culture, the cells displayed the typical 'hill and valley' growth pattern (Fig. 1F), and were positive for $\alpha$-SMA, according to immunofluorescent staining (Fig. 1G).

MSC-CM improves $P H$. The examination of hemodynamics 21 days after injection of MCT indicated a significant increase in RVSP compared with the control group $(48.26 \pm 3.93$ vs. $27.58 \pm 3.47 \mathrm{mmHg} ; \mathrm{P}<0.01$; Fig. $2 \mathrm{~A}$ ), and a significant decrease in MAoP compared with the control group $(89.54 \pm 15.81$ vs. $116.37 \pm 16.17 \mathrm{mmHg}$; $\mathrm{P}<0.05$; Fig. $2 \mathrm{~B})$. In accordance with this finding, histological examination also indicated that, 21 days after injection with MCT, medial hypertrophy of pulmonary muscular arterioles was evident in the model group (Fig. 2B). Additionally, WT levels were significantly increased in the model group compared with the control $(34.12 \pm 3.59$ vs. $12.53 \pm 1.82 \%$; P $<0.01$; Fig. 2 C). These changes indicated the development of $\mathrm{PH}$ in the experimental rat.

Administration of MSC-CM leads to the improvement of abnormal hemodynamics. RVSP decreased to $31.42 \pm 3.38 \mathrm{mmHg}$ (Fig. 2A) and MAoP increased to $112.97 \pm 14.26 \mathrm{mmHg}$ (Fig. 2B) following MSC-CM treatment; these values were significantly different compared with the model group $(\mathrm{P}<0.05)$. In addition, the medial hypertrophy of pulmonary muscular arterioles was improved (Fig. 2C), and the WT was significantly decreased $(13.28 \pm 1.78 \%)$ compared with that in the model group $(34.12 \pm 3.59 \%$; $\mathrm{P}<0.01$; Fig. $2 \mathrm{C})$.

MSC-CM decreases the protein expression levels of TNF- $\alpha$ in lung tissue. Compared with the control group (Fig. 3A), immunohistochemical staining revealed that an abundance of cells were positive for TNF- $\alpha$ within the lung tissue 21 days after injection of MCT in the model group (Fig. 3B). Following the administration of MSC-CM, the number of cells positive for TNF- $\alpha$ decreased (Fig. 3C), and the average OD significantly decreased from $0.23 \pm 0.07$ in the model group to $0.17 \pm 0.06$ in the MSC-CM treatment group $(\mathrm{P}<0.01$; Fig. 3D).
MSC-CM suppresses the production of TNF- $\alpha$ and proliferation of PASMCs in co-culture systems. After 3 days of culture, levels of TNF- $\alpha$ in the supernatant of the co-culture system were analyzed by ELISA. The results revealed that high levels of TNF- $\alpha(762.51 \pm 42.35 \mathrm{pg} / \mathrm{ml})$ were produced by $\mathrm{T}$ cells under stimulation with ConA (Fig. 1A). However, in the presence of MSC-CM, the production of TNF- $\alpha$ was significantly inhibited $(218.25 \pm 19.33 \mathrm{pg} / \mathrm{ml} ; \mathrm{P}<0.01$; Fig. 4A). Additionally, the proliferation of PASMCs, as assessed by an MTS assay, revealed that the proliferation of PASMCs significantly increased when co-cultured with ConA-stimulated $\mathrm{T}$ cells, compared with PASMCs cultured alone $(0.94 \pm 0.05$ vs. $0.73 \pm 0.06$; $\mathrm{P}<0.01$; Fig. $4 \mathrm{~B})$. Furthermore, the ability of $\mathrm{T}$ cells in promoting the proliferation of PASMCs was significantly inhibited by treatment with MSC-CM $(0.78 \pm 0.07$; $\mathrm{P}<0.01$; Fig. 4B).

MSC-CM downregulates the expression of CaN and $N F A T c 2 . \mathrm{CaN}$ and NFATc2 are critical regulatory factors of SMC proliferation, thus, the expression levels of both were assessed by reverse transcription-quantitative polymerase chain reaction (11). The results indicated that, after 3 days of co-culture with ConA-stimulated T cells, the expression levels of CaN and NFATc2 in PASMCs were significantly upregulated compared with the expression in PASMCs alone. Furthermore, MSC-CM was able to significantly reduce the expression levels of $\mathrm{CaN}$ and NFATc2 $(\mathrm{P}<0.01)$ that were increased by the promoting effects of the activated $\mathrm{T}$ cells (Fig. 4C and D).

MSC-CM suppresses CaN activity and NFATc2 activation in PASMCs. In the co-culture system, it was observed that intracellular $\mathrm{CaN}$ activity was significantly upregulated in PASMCs cultured for 3 days with ConA-stimulated T cells, compared with PASMCs cultured alone $(0.59 \pm 0.06$ vs. $0.32 \pm 0.04 \mathrm{U} / \mathrm{mgprot} ; \mathrm{P}<0.01$; Fig. 4E). Furthermore, the majority of NFATc2 was translocated to the nucleus, indicating the activation of NFATc2 (Fig. 4G). Following the addition of MSC-CM, the intracellular CaN activity of PASMCs was significantly decreased $(0.37 \pm 0.06 \mathrm{U} / \mathrm{mgprot} ; \mathrm{P}<0.01 ;$ Fig. $4 \mathrm{E})$. When PASMCs were cultured independently, the expression of NFATc2 was predominantly localized to the cytoplasm (Fig. 4F). Finally, addition of MSC-CM also led to suppression of NFATc2 activation (Fig. 4H).

\section{Discussion}

The present study demonstrated that the administration of MSC-CM decreases protein expression levels of TNF- $\alpha$ in the lungs of rat models with MCT-induced PH. In addition, improvements in hemodynamic and histological abnormalities were also observed. A co-culture system comprised of activated T cells and PASMCs was established to further reveal the therapeutic mechanism of MSC-CM in PH. The in vitro co-culture system revealed that the high levels of TNF- $\alpha$ produced as a result of activated T cells leads to the upregulation of $\mathrm{CaN}$ and NFATc2 expression. Additional effects observed included an increase in intracellular $\mathrm{CaN}$ activity and NFATc2 activation in PASMCs, thus promoting the proliferation of PASMCs. MSC-CM was able to effectively 
A

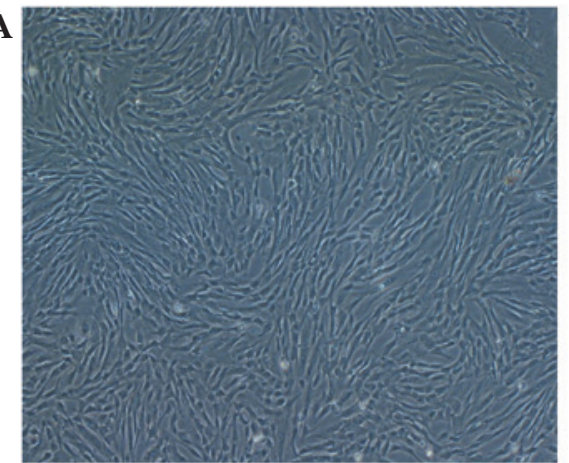

D
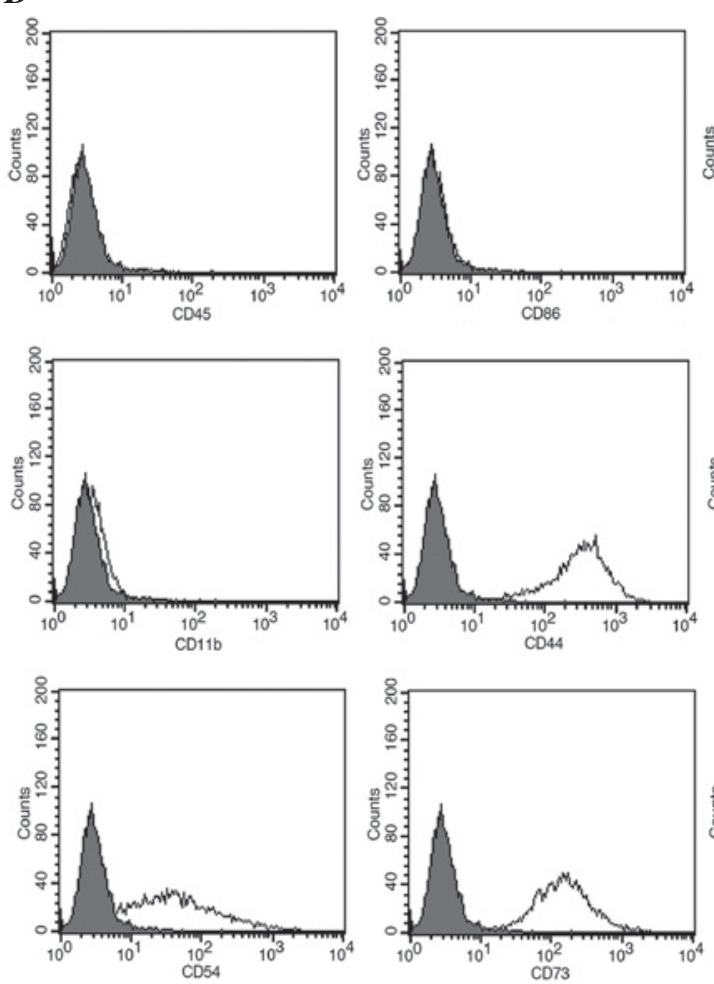
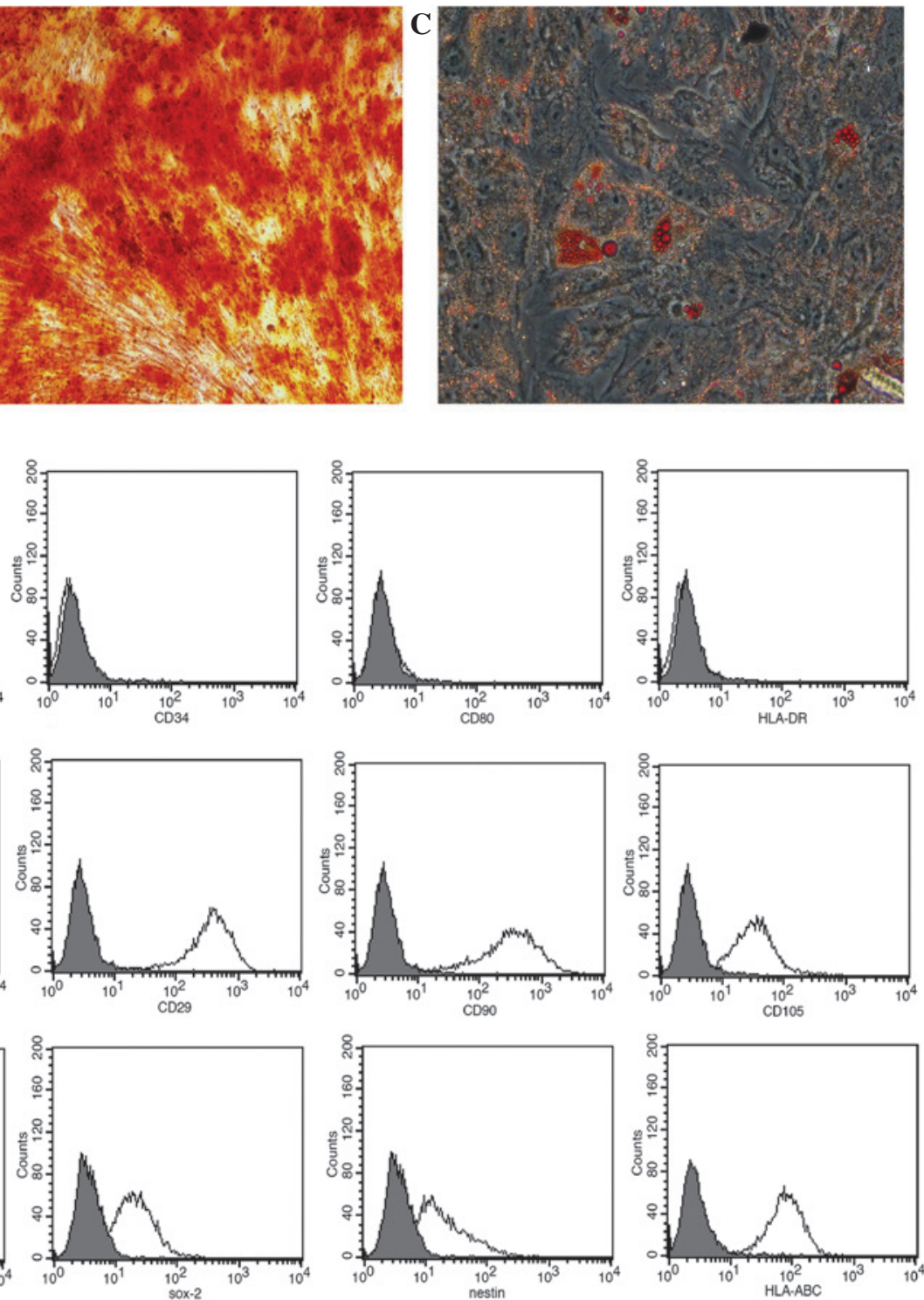
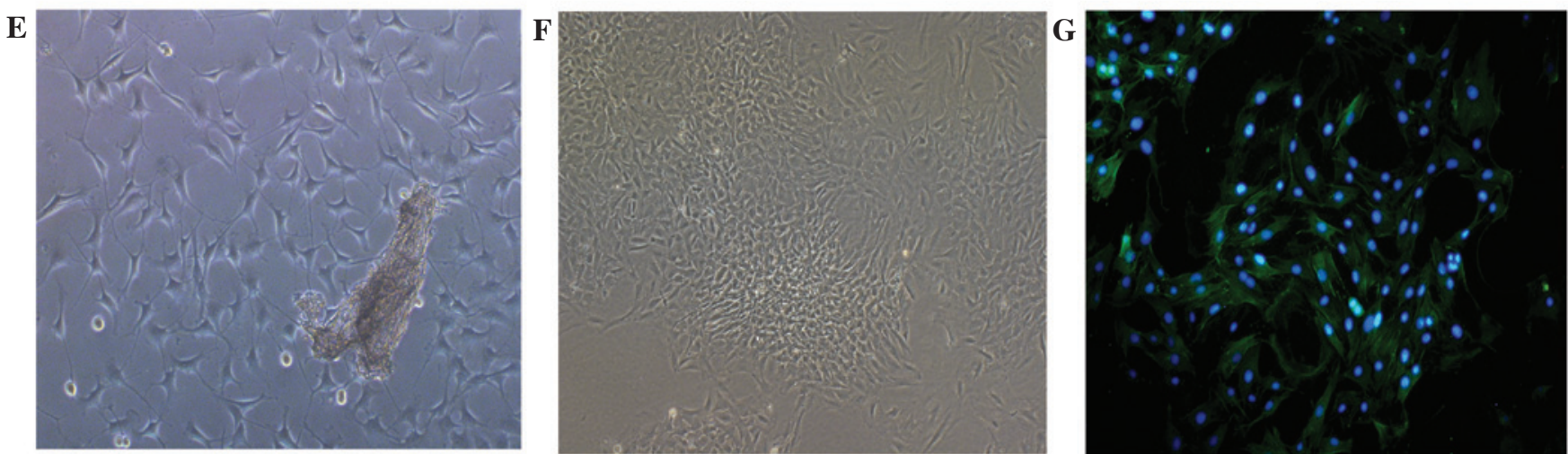

Figure 1. Growth of mesenchymal stem cells (MSCs) and pulmonary artery smooth muscle cells (PASMCs) in vitro. (A) MSCs culured in serum-free medium at passage 3 (magnification, x100). (B) Osteogenic and (C) adipogenic differentiation of MSCs cultured in MSC-conditioned media identified using Alizarin red S or Oil Red O stain, respectively (magnification, x200). (D) Phenotypic analysis of MSCs, assessed using flow cytometry. (E) PASMC tissue explant cultured for 5 days (magnification, x100). (F) PASMCs at passage 3 (magnification, x100). (G) PASMC immunofluorescent staining for $\alpha$-smooth muscle actin (magnification, x200). CD, cluster of differentiation; Sox-2, SRY box-2; HLA, human leukocyte antigen.

suppress the production of high levels of TNF- $\alpha$ by T cells, which led to the downregulation of expression levels of $\mathrm{CaN}$ and NFATc2 in PASMCs. This had the effect of decreasing
CaN activity and preventing NFATc2 activation, which consequently resulted in suppression of the proliferation of PASMCs. 
A

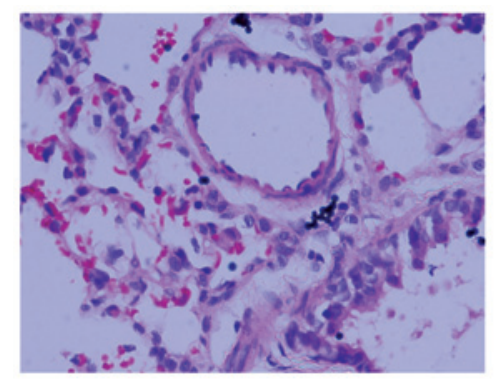

B

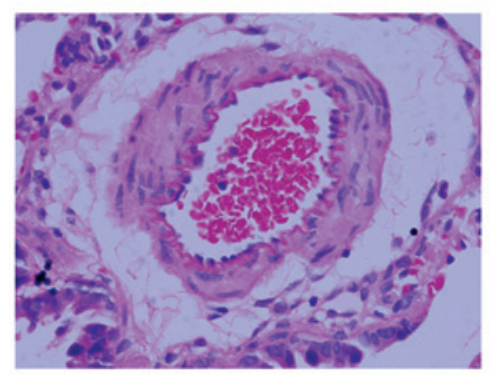

C

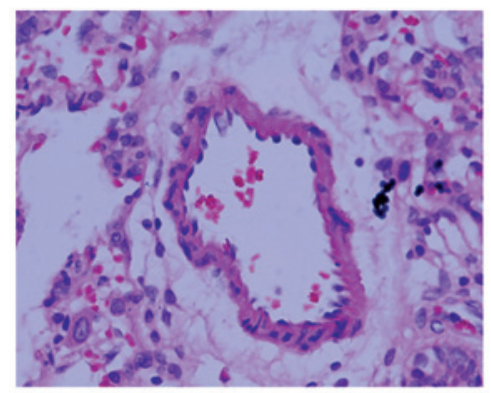

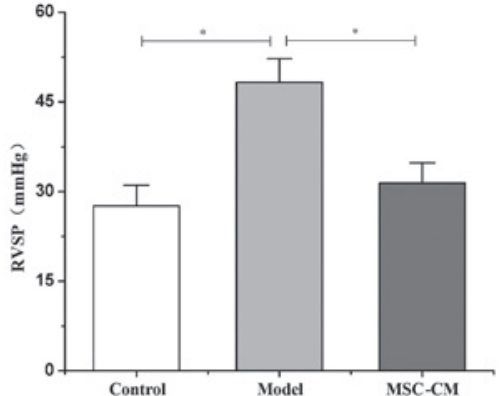
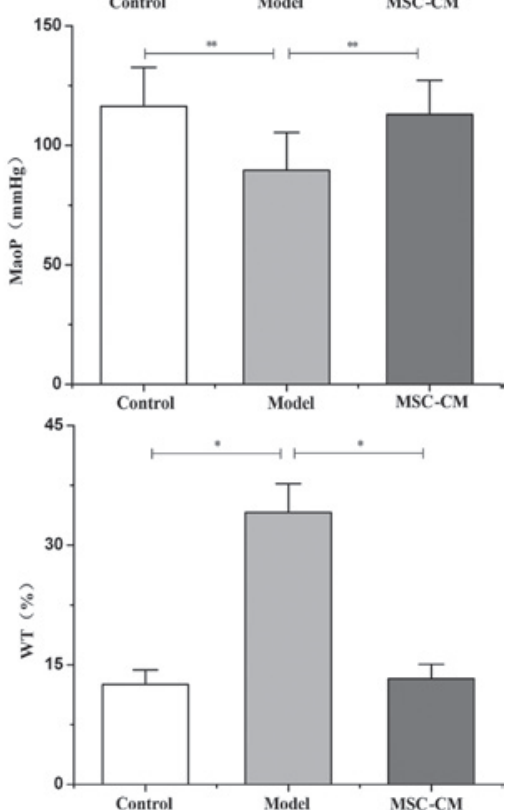

Figure 2. Effects of MSC-CM on monocrotaline-induced pulmonary hypertension. Left panels: Histological examination of lung tissue in (A) control, (B) model and (C) MSC-CM treatment groups (hematoxylin and eosin stain; magnification, x400). Right panels: Changes of (A) RVSP, (B) MAoP and (C) medial WT in each group. Data are presented as mean \pm standard deviation $(n=6) .{ }^{*} \mathrm{P}<0.01,{ }^{* * *} \mathrm{P}<0.05$. RVSP, right ventricle systolic pressure; MSC-CM, mesenchymal stem cell-conditioned media; MAoP, mean aortic pressure; WT, wall thickness.

A

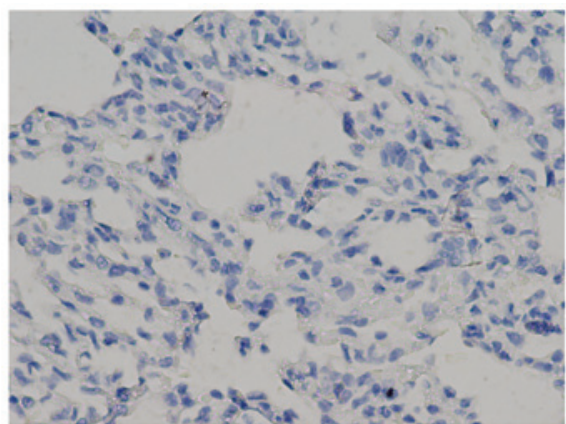

$\mathbf{C}$

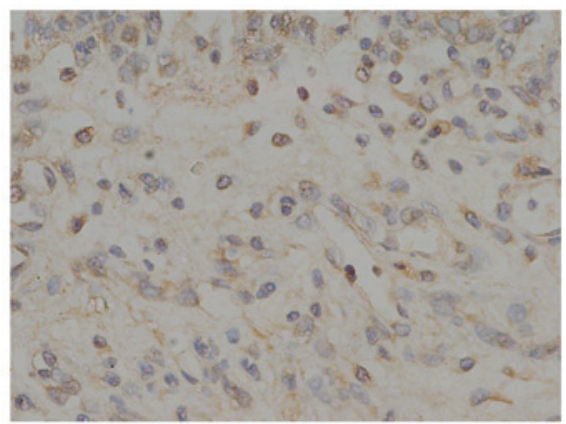

B

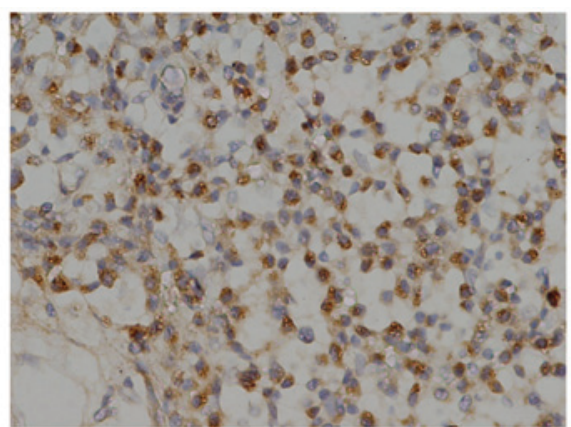

D

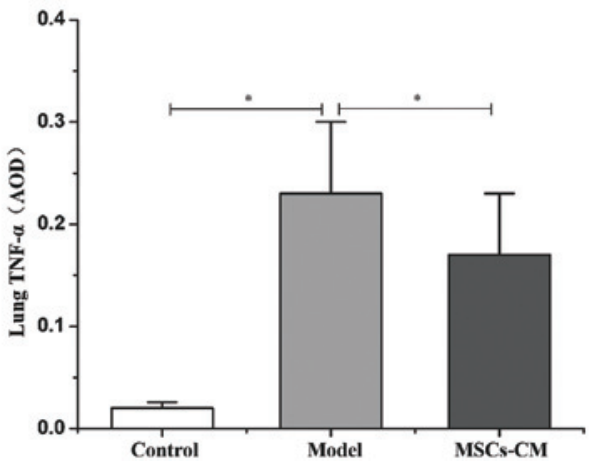

Figure 3. Protein expression levels of TNF- $\alpha$ in lung tissue. Immunohistochemical staining for TNF- $\alpha$ in the (A) control, (B) model and (C) MSC-CM treatment groups (3-amino-9-ethylcarbazole peroxidase staining; magnification, $\mathrm{x} 400$ ). (D) Quantified expression levels of TNF- $\alpha$ in lung tissue. Data are presented as mean \pm standard deviation $(\mathrm{n}=6)$. " $\mathrm{P}<0.01$. TNF- $\alpha$, tumor necrosis factor- $\alpha$; MSC-CM, mesenchymal stem cell-conditioned media; AOD, average optical density. 

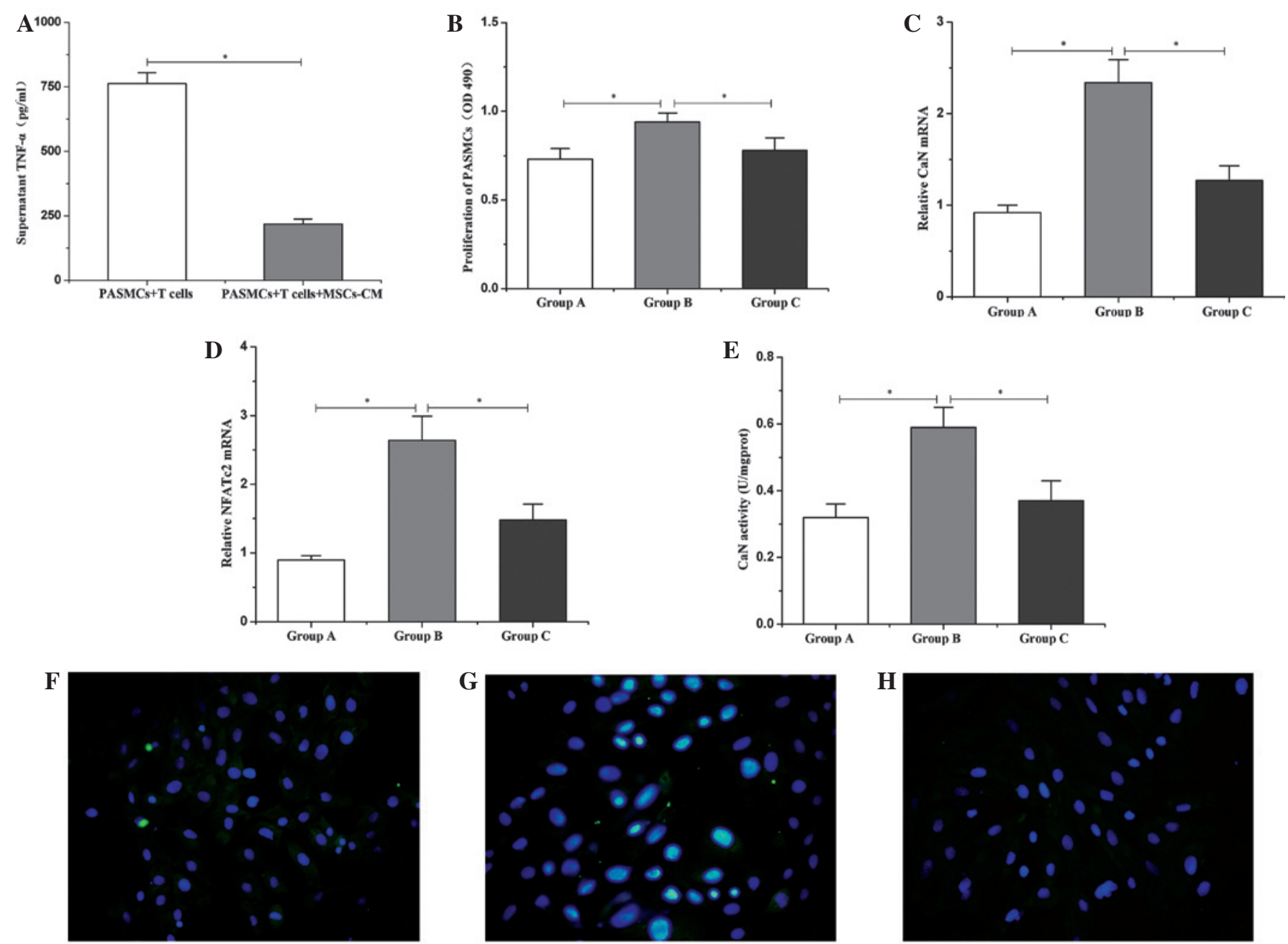

Figure 4. Changes in TNF- $\alpha$, CaN and NFATc2 in the co-culture system. (A) Protein expression levels of TNF- $\alpha$ in the supernatant. (B) Proliferation of PASMCs. mRNA expression of (C) CaN and (D) NFATc2 in PASMCs. (E) CaN activity in PASMCs. Group A, PASMCs alone; Group B, PASMCs + T cells; Group C, PASMCs + T cells + MSC-CM. Data are presented as mean \pm standard deviation $(n=6)$. ${ }^{*} P<0.01$. Activation of NFATc2 in PASMCs in the co-culture system for (F) group A; (G) group B, and (H) group C (fluorescein isothiocyanate/DAPI staining; magnification, $\mathrm{x} 400$ ). TNF- $\alpha$, tumor necrosis factor- $\alpha$; PASMCs, pulmonary artery smooth muscle cells; MSC-CM, mesenchymal stem cell-conditioned media; OD, optical density; CaN, calcineurin; NFATc2, nuclear factor of activated T cells c2.

Persistent contraction and hypertrophy of pulmonary arterioles are considered to be defining characteristics of PH (24). Inflammation and the overproliferation of PASMCs are also hypothesized to have an involvement in the pathological process, thus rendering them a therapeutic target for the treatment of PH (12). MSCs, as a subset of stromal stem cells, have attracted much attention as potential therapeutic vehicles for the treatment of $\mathrm{PH}$ and other diseases of the lung, due to their potent immunomodulatory, anti-inflammatory and anti-fibrotic properties (25-27). Previously, it has been demonstrated that MSCs have the ability to suppress activated $\mathrm{CD}^{+} \mathrm{T}$ cells, thereby preventing the production of inflammatory cytokines when co-cultured (14). Furthermore, the immunosuppressive activity of MSCs is highly associated with the secretion of PGE2 and immunoregulatory cytokines (14). Due to the extensive level of inflammation involved in the pathological mechanism of $\mathrm{PH}$, the immunosuppressive effects of MSCs may be beneficial to the treatment of $\mathrm{PH}$. The exact mechanism detailing the involvement of MSCs in PH, however, remains unclear. A former hypothesis suggesting that MSCs had the ability to differentiate into various lung tissue types following transplantation has been somewhat discredited due to the existence of the MSC niche, which may serve to hinder the homing and differentiation of MSCs (28). Furthermore, due to the ability of MSC-CM to attenuate hypoxia-induced pulmonary injury (15), the paracrine mechanism is generally considered to have greater plausibility in explaining the protective effects of MSCs. With the additional benefit of no ethical restrictions against its use, MSC-CM, which possesses numerous therapeutic effects, may be a promising vehicle in the clinic. In the present study, a rat model of MCT-induced $\mathrm{PH}$ was used to assess the immunoregulatory capabilities and therapeutic effects of MSC-CM. The results demonstrated that expression levels of TNF- $\alpha$ within the lung tissue were decreased by the immunosuppressive activity of MSC-CM. As a result, hemodynamic abnormalities, including increased RVSP and decreased MAoP, were improved and approaching normal levels. Further examination of the hemodynamics also indicated that the medial hypertrophy and remodeling of pulmonary arterioles were prevented. These results may indicate the mechanisms by which the immunoregulatory 
properties of MSC-CM are responsible for potential therapeutic benefits in the treatment of $\mathrm{PH}$.

The NFAT family of proteins contains a series of pivotal regulators of immune response that are widely expressed in a host of tissues and organs (29), including smooth muscle cells (30). Typically, NFAT proteins are predominately distributed in the cytoplasm, where they are inactive. Upon dephosphorylation by the $\mathrm{Ca}^{2+}$-responsive phosphatase $\mathrm{CaN}$, NFAT proteins are activated and translocated to the nucleus in order to exert their regulatory actions (29). It has previously been identified that the activation of CaN and NFAT are involved in the pathogenesis of PH (9).

NFAT activation has been observed in PASCs obtained from patients with $\mathrm{PH}$ (12) and in experimental animal models, such as MCT-induced (12) and hypoxia-induced (13) PH models. Furthermore, inhibition of CaN or NFAT may result in a decrease in the proliferation of PASMCs, and an increase the levels of apoptosis (12), thereby inhibiting hypoxia-induced $\mathrm{PH}$ and right ventricular hypertrophy (31). In order to further elucidate the exact therapeutic mechanism of MSC-CM in $\mathrm{PH}$, the present study used an in vitro co-culture system containing $\mathrm{T}$ cells and PASMCs. The supernatant protein expression levels of TNF- $\alpha$, mRNA expression levels of CaN and NFAT, as well as CaN activity and NFATc2 activation in PASMCs were assessed to better understand the mechanisms pertaining to the regulatory effects of MSC-CM on the inflammation-associated overproliferation of PASMCs. The results indicated that, under the stimulation of ConA, T cells produced high levels of TNF- $\alpha$, resulting in the upregulation of $\mathrm{CaN}$ and NFAT mRNA expression levels, increased $\mathrm{CaN}$ activity, and promoted activation of NFATc2, thus accelerating the proliferation of PASMCs. As a result of the immunosuppressive effects of MSC-CM, TNF- $\alpha$ production levels significantly decreased. This immunosuppressive effect was also associated with downregulated expression levels of CaN and NFAT in PASMCs; in agreement, $\mathrm{CaN}$ activity and NFATc2 activation levels were observed to have decreased. Consequentially, the overproliferation of PASMCs was effectively suppressed by MSC-CM.

In conclusion, the present study provides new evidence highlighting the ability of conditioned media from MSCs to suppress the overproliferation of PASMCs, and thus have possible therapeutic benefits in the treatment of $\mathrm{PH}$. The predominant reason for this effect was a result of the suppression of TNF- $\alpha$, and the subsequent inhibitory action on the expression levels of $\mathrm{CaN}$ and NFAT, CaN activity, and the activation of NFATc2 in PASMCs. However, the long-term impacts and potential side-effects of this prospective treatment require further investigation.

\section{Acknowledgements}

The present study was supported by the National Natural Science Foundation of China (grant nos. 30560159, 30960412 and 31360285).

\section{References}

1. Bazan IS and Fares WH: Pulmonary hypertension: Diagnostic and therapeutic challenges. Ther Clin Risk Manag 11: 1221-1233, 2015 .

2. Tonelli AR: Pulmonary hypertension survival effects and treatment options in cystic fibrosis. Curr Opin Pulm Med 19: 652-661, 2013.
3. Tonelli AR, Fernandez-Bussy S, Lodhi S, Akindipe OA, Carrie RD, Hamilton K, Mubarak K and Baz MA: Prevalence of pulmonary hypertension in end-stage cystic fibrosis and correlation with survival. J Heart Lung Transplant 29: 865-872, 2010.

4. Hayes D Jr, Higgins RS, Kirkby S, McCoy KS, Wehr AM, Lehman AM and Whitson BA: Impact of pulmonary hypertension on survival in patients with cystic fibrosis undergoing lung transplantation: An analysis of the UNOS registry. J Cyst Fibros 13: 416-423, 2014

5. Pinto RF, Higuchi Mde L and Aiello VD: Decreased numbers of T-lymphocytes and predominance of recently recruited macrophages in the walls of peripheral pulmonary arteries from 26 patients with pulmonary hypertension secondary to congenital cardiac shunts. Cardiovasc Pathol 13: 268-275, 2004.

6. Perros F, Dorfmüller P, Montani D, Hammad H, Waelput W, Girerd B, Raymond N, Mercier O, Mussot S, Cohen-Kaminsky S, et al: Pulmonary lymphoid neogenesis in idiopathic pulmonary arterial hypertension. Am J Respir Crit Care Med 185: 311-321, 2012

7. Heath D and Edwards JE: The pathology of hypertensive pulmonary vascular disease; a description of six grades of structural changes in the pulmonary arteries with special reference to congenital cardiac septal defects. Circulation 18: 533-547, 1958.

8. Soon E, Holmes AM, Treacy CM, Doughty NJ, Southgate L, Machado RD, Trembath RC, Jennings S, Barker L, Nicklin P, et al: Elevated levels of inflammatory cytokines predict survival in idiopathic and familial pulmonary arterial hypertension. Circulation 122: 920-927, 2010.

9. Frid MG, Brunetti JA, Burke DL, Carpenter TC, Davie NJ, Reeves JT, Roedersheimer MT, van Rooijen N and Stenmark KR: Hypoxia-induced pulmonary vascular remodeling requires recruitment of circulating mesenchymal precursors of a monocyte/macrophage lineage. Am J Pathol 168: 659-669, 2006.

10. Rowlands DJ, Islam MN, Das SR, Huertas A, Quadri SK, Horiuchi K, Inamdar N, Emin MT, Lindert J, Ten VS, et al: Activation of TNFR1 ectodomain shedding by mitochondrial $\mathrm{Ca}^{2+}$ determines the severity of inflammation in mouse lung microvessels. J Clin Invest 121: 1986-1999, 2011.

11. Said SI, Hamidi SA and Gonzalez Bosc L: Asthma and pulmonary arterial hypertension: Do they share a key mechanism of pathogenesis? Eur Respir J 35: 730-734, 2010.

12. Bonnet S, Rochefort G, Sutendra G, Archer SL, Haromy A, Webster L, Hashimoto K, Bonnet SN and Michelakis ED: The nuclear factor of activated $\mathrm{T}$ cells in pulmonary arterial hypertension can be therapeutically targeted. Proc Natl Acad Sci USA 104: 11418-11423, 2007.

13. de Frutos S, Spangler R, Alò D and Bosc LV: NFATc3 mediates chronic hypoxia-induced pulmonary arterial remodeling with alpha-actin up-regulation. J Biol Chem 282: 15081-15089, 2007.

14. Yang ZX, Han ZB, Ji YR, Wang YW, Liang L, Chi Y, Yang SG, Li LN, Luo WF, Li JP, et al: CD106 identifies a subpopulation of mesenchymal stem cells with unique immunomodulatory properties. PLoS One 8: e59354, 2013.

15. Aslam M, Baveja R, Liang OD, Fernandez-Gonzalez A, Lee C, Mitsialis SA and Kourembanas S: Bone marrow stromal cells attenuate lung injury in a murine model of neonatal chronic lung disease. Am J Respir Crit Care Med 180: 1122-1130, 2009.

16. Lu LL, Liu YJ, Yang SG, Zhao QJ, Wang X, Gong W, Han ZB, Xu ZS, Lu YX, Liu D, et al: Isolation and characterization of human umbilical cord mesenchymal stem cells with hematopoiesis-supportive function and other potentials. Haematologica 91: 1017-1026, 2006.

17. Matsuda Y, Hoshikawa Y, Ameshima S, Suzuki S, Okada Y, Tabata T, Sugawara T, Matsumura Y and Kondo T: Effects of peroxisome proliferator-activated receptor gamma ligands on monocrotaline-induced pulmonary hypertension in rats. Nihon Kokyuki Gakkai Zasshi 43: 283-288, 2005 (In Japanese).

18. Liu JF, DU ZD, Chen Z, Han ZC and He ZX: Granulocyte colony-stimulating factor attenuates monocrotaline-induced pulmonary hypertension by upregulating endothelial progenitor cells via the nitric oxide system. Exp Ther Med 6: 1402-1408, 2013.

19. Maruyama H, Watanabe S, Kimura T, Liang J, Nagasawa T, Onodera M, Aonuma K and Yamaguchi I: Granulocyte colony-stimulating factor prevents progression of monocrotaline-induced pulmonary arterial hypertension in rats. Circ J 71: 138-143, 2007.

20. Woodrum DT, Ford JW, Cho BS, Hannawa KK, Stanley JC, Henke PK and Upchurch GR Jr: Differential effect of 17-beta-estradiol on smooth muscle cell and aortic explant MMP2. J Surg Res 155: 48-53, 2009. 
21. Ahmad S, Choudhry MA, Shankar R and Sayeed MM: Transforming growth factor-beta negatively modulates T-cell responses in sepsis. FEBS Lett 402: 213-218, 1997.

22. Liu JF, Han ZB, Han ZC and He ZX: Mesenchymal stem cells suppress CaN/NFAT expression in the pulmonary arteries of rats with pulmonary hypertension. Exp Ther Med 10: 1657-1664, 2015.

23. Livak KJ and Schmittgen TD: Analysis of relative gene expression data using real-time quantitative PCR and the 2(-Delta Delta C(T)) Method. Methods 25: 402-408, 2001.

24. Tuder RM, Marecki JC, Richter A, Fijalkowska I and Flores S: Pathology of pulmonary hypertension. Clin Chest Med 28: 23-42, 2007.

25. Weiss DJ, Bertoncello I, Borok Z,Kim C, Panoskaltsis-Mortari A, Reynolds S, Rojas M, Stripp B, Warburton D and Prockop DJ: Stem cells and cell therapies in lung biology and lung diseases. Proc Am Thorac Soc 8: 223-72, 2011.

26. OrtizLA,GambelliF,McBrideC,Gaupp D, Baddoo M,KaminskiN and Phinney DG: Mesenchymal stem cell engraftment in lung is enhanced in response to bleomycin exposure and ameliorates its fibrotic effects. Proc Natl Acad Sci USA 100: 8407-8411, 2003.
27. Hansmann G, Fernandez-Gonzalez A, Aslam M, Vitali SH, Martin T, Mitsialis SA and Kourembanas S: Mesenchymal stem cell-mediated reversal of bronchopulmonary dysplasia and associated pulmonary hypertension. Pulm Circ 2: 170-81, 2012.

28. Jun D, Garat C, West J, Thorn N, Chow K, Cleaver T, Sullivan T, Torchia EC, Childs C, Shade T, et al: The pathology of bleomycin-induced fibrosis is associated with loss of resident lung mesenchymal stem cells that regulate effector T cell proliferation. Stem Cells 29: 725-735, 2011.

29. Pan MG, Xiong Y and Chen F: NFAT gene family in inflammation and cancer. Curr Mol Med 13: 543-554, 2013.

30. Kudryavtseva O, Aalkjaer C and Matchkov VV: Vascular smooth muscle cell phenotype is defined by $\mathrm{Ca}^{2+}$-dependent transcription factors. FEBS J 280: 5488-5499, 2013.

31. Koulmann N, Novel-Chaté V, Peinnequin A, Chapot R, Serrurier B, Simler N, Richard H, Ventura-Clapier R and Bigard X: Cyclosporin A inhibits hypoxia-induced pulmonary hypertension and right ventricle hypertrophy. Am J Respir Crit Care Med 174: 699-705, 2006. 